\title{
OPEN The change of gut microbiota in MDD patients under SSRIs treatment
}

\author{
Yang Shen ${ }^{1,5}$, Xiao Yang ${ }^{2,5}$, Gaofei $\mathrm{Li}^{3,5}$, Jiayu Gao ${ }^{4 \bowtie}$ \& Ying Liang ${ }^{1 \bowtie}$
}

The alterations in the gut microbiota have been reported to be correlated with the development of depression. The purpose of this study was to investigate the changes of intestinal microbiota in depressed patients after antidepressant treatment. We recruited 30 MDD patients (MDD group) and 30 healthy controls (control group). The MDD group received individualized treatment with escitalopram at a maximum dose of $20 \mathrm{mg} /$ day. After depressive symptoms improved to a HAMD scale score $>50 \%$, a fecal sample was collected again and used as the follow-up group. The differences of gut microbiota between patients and controls, the characteristics of gut microbiota under treatment and the potential differences in metabolic functions were thus investigated. The Firmicutes/Bacteroidetes ratio was significantly different within three groups, and the ratio of follow-up group was significantly lower than those of the other two groups. Alpha diversity was significantly higher in MDD group than those of the other groups, and the alpha diversity was not significantly different between control and follow-up groups. The beta diversity of some patients resembled participants in the control group. The metabolic function of gut microbiota after treatment was still different from that of the control group. This study suggests that the intestinal flora of depressed patients has a tendency to return to normal under escitalopram treatment.

As a common mental disorder accompanied by high disability and suicide, major depressive disorder (MDD) has become a worldwide issue ${ }^{1}$. In recent years, several studies have shown the correlation between gut microbiota and the development of depression ${ }^{2-4}$. In several studies, MDD patients showed specific features of gut microbes differing from normal controls ${ }^{5-8}$. It has been reported that transplants of MDD patients' feces into mice could cause the depression-like behaviors, which used to establish animal models of $\mathrm{MDD}^{5,9,10}$. Moreover, it also observed that the fecal bacteria transplantation of patients could lead to the increase of microglial cell density and expression of IL- 1 in the ventral hippocampus ${ }^{11}$. Taken together, the change of gut microbiota could be correlated with the occurrence of MDD.

At present, selective serotonin reuptake inhibitors (SSRIs) are widely used in clinical practice and have therapeutic effects in the treatment of depression ${ }^{12}$. Besides, several SSRIs drugs, including sertraline, fluoxetine, paroxetine and escitalopram, could present antibacterial effects directly ${ }^{13,14}$. For example, staphylococcus and enterococcus are especially vulnerable to sertraline, fluoxetine and paroxetine ${ }^{14-16}$. Therefore, SSRIs have demonstrated both of antidepressant and antimicrobial properties ${ }^{17}$. Ramsteijn et al.s study reported that fluoxetine treatment altered important features of this transition from pregnancy to lactation and led to the decreased fecal amino acid concentrations. Amino acid concentrations negatively correlated with the relative abundance of bacterial taxa such as Prevotella and Bacteroides ${ }^{18}$. McVey Neufeld et al. reported that intestinal exposure to SSRIs could increase the excitability of intrinsic primary afferent neurons in the intermuscle plexus and alter the alpha diversity of the intestinal microbiota ${ }^{19}$.

Overall, SSRIs could directly or indirectly influence the changes of gut microbiota which might play the key role in the development of MDD. The purpose of this study was to explore the difference of gut microbiota with first episode MDD and elucidate the changes of gut microbiota after treatment by SSRIs.

\footnotetext{
${ }^{1}$ National Clinical Research Center for Mental Disorders, Peking University Sixth Hospital, Institute of Mental Health, Key Laboratory of Mental Health, Ministry of Health, Peking University, Haidian District, Huayuanbeilu 51, Beijing 100191, China. 'School of Clinical Medicine, Henan University of Science and Technology, Luoyang, China. ${ }^{3}$ Department of Psychiatry, Beijing Hospital of Chinese Traditional and Western Medicine, Beijing, China. ${ }^{4}$ School of Chemical Engineering and Pharmaceutics, Henan University of Science and Technology, No. 263, Kaiyuan Boulevard, Luolong District, Luoyang 471023, Henan, China. ${ }^{5}$ These authors contributed equally: Yang Shen, Xiao Yang and Gaofei Li. ${ }^{\bowtie}$ email: j.gao@haust.edu.cn; liangying1980@bjmu.edu.cn
} 


\begin{tabular}{|l|l|l|l|}
\hline & MDD $(\mathbf{n}=30)$ & Controls $(\mathbf{n}=30)$ & \multirow{2}{*}{} \\
\cline { 2 - 3 } & $\mathbf{M} \pm$ value & $\mathbf{M} \pm$ SD & 0.757 \\
\hline Age (years) & $44.83 \pm 11.00$ & $43.97 \pm 10.57$ & 0.605 \\
\hline Gender $(\mathrm{M} / \mathrm{F})$ & $13 / 17$ & $15 / 15$ & 0.171 \\
\hline Height $(\mathrm{m})$ & $1.68 \pm 0.07$ & $1.70 \pm 0.05$ & 0.447 \\
\hline Weight $(\mathrm{kg})$ & $67.83 \pm 6.86$ & $69.21 \pm 7.14$ & 0.761 \\
\hline BMI $\left(\mathrm{kg} / \mathrm{m}^{2}\right)$ & $23.99 \pm 2.05$ & $23.83 \pm 2.08$ & 0.288 \\
\hline Tobacco $(\%)^{\mathrm{a}}$ & $46.67 \%$ & $30.00 \%$ & 0.192 \\
\hline Alcohol $(\%)^{\mathrm{a}}$ & $53.33 \%$ & $33.33 \%$ & \multicolumn{2}{|l}{} \\
\hline
\end{tabular}

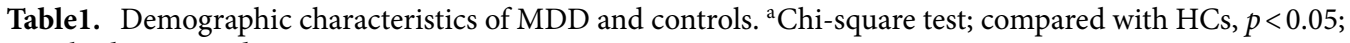
$B M I$ body mass index.

a

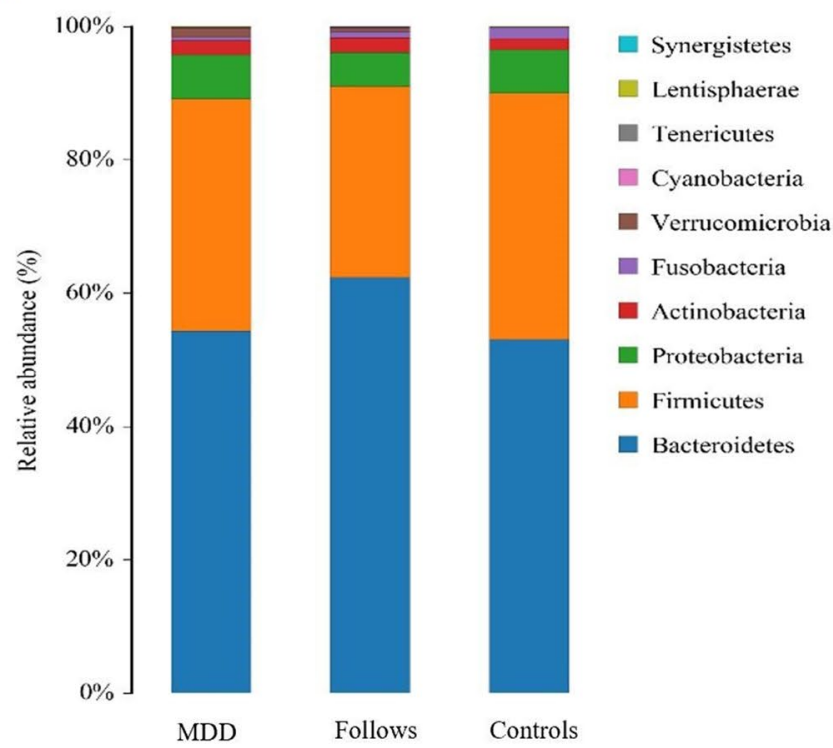

b

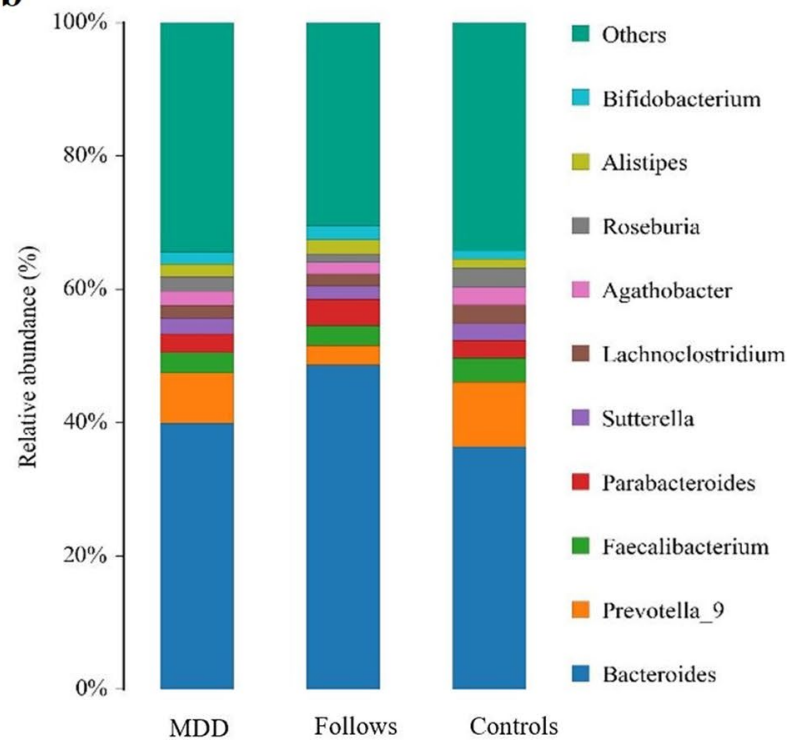

Figure 1. Histogram of species distribution. QIIME software was used to generate species abundance tables at different taxonomic levels, and $\mathrm{R}$ language tool was used to draw community structure charts at different taxonomic levels. (a) Relative proportions of species distribution at the phylum level; (b) relative proportions of species distribution at the genus level.

\section{Results}

Clinical data. In this study, 30 patients with drug-naive first-episode MDD and 30 healthy controls were recruited, respectively. There were no statistically significant differences between patients' group and controls group in terms of age, gender, height, weight, and tobacco and alcohol consumption $(p>0.05)$ (Table 1). The MDD group received an individualized treatment and the maximum dose was $20 \mathrm{mg} /$ day. The average dose of escitalopram was $16.33 \pm 3.46 \mathrm{mg} /$ day. Under escitalopram treatment, the mean time of the HAMD score decreased over $50 \%$ was $34.53 \pm 5.18$ days. All patients have response with $50 \%$ HAMD reduction.

Sequencing data and bacterial taxonomic composition. Sequencing data. Total 4,790,651 original sequences were obtained from 90 samples. After double-end Reads splicing and filtering, a total of 4,444,748 Clean tags were generated. Each sample generated at least 12,039 Clean tags. Taxonomic annotation of OTUs was based on Silva (version 138.1) and UNITE (version 7.0) taxonomic databases.

Bacterial composition comparisons within three groups. At phylum level, the dominated gut microbiota was composed by Bacteroidetes, Firmicutes, Proteobacteria and Actinobacteria in three groups. Bacteroidetes and Firmicutes accounted for nearly $90 \%$ of the total gut microbiota. At the genus level, the distribution proportion of several gut microbiota abundance in the three groups was statistically different $(q<0.05)$ (Supplementary Table S1). Through the calculation, the Firmicutes/Bacteroidetes ratio of MDD group, Follow-up group and Controls group were $0.64,0.46$, and 0.70 , respectively. The ratio in Follow-up group was significantly lower than those of the other two groups. There were significant differences among the three groups $(p<0.05)$ (Fig. 1). 
Bacterial composition comparisons before treatment. Between the MDD and Controls groups, there were significant differences in the abundance of multiple gut microbiota at the genus level. The abundance of Parasutterella, Prevotella_9, Fusobacterium, Prevotella_2, Christensenellaceae_R-7_group, Odoribacter and [Eubacterium] _ruminantium_group significantly decreased in MDD group. Meanwhile, The abundance of Parabacteroides, Lactobacillus, Anaerostipes, and Ruminococcaceae_UCG-014 significantly increased in MDD group $(q<0.05)$ (Supplementary Table S2).

Bacterial composition comparisons after treatment. After escitalopram treatment, the abundance of Christensenellaceae_R-7_group, [Eubacterium]_ruminantium_group and Fusobacterium significantly increased in Follow-up group $(q<0.05)$. The abundance of Lactobacillus significantly decreased in the Followup group $(q<0.05)$. The main change of gut microbiota abundance in Follow-up group was Bacteroides (Supplementary Table S2).

In addition, there were also several differences in the gut microbiota between Follow-up group and Controls group. In Follow-up group, the abundance of Parabacteroides, Prevotellaceae, Ruminiclostridium_6, Flavonifractor significantly increased $(q<0.05)$, while that of Prevotella_2, Lachnospira, Collinsella, and Clostridium_ sensu_stricto_1 significantly decreased $(q<0.05)$. Moreover, the abundance of Faecalibacterium and Lachnoclostridium in Follow-up group or in MDD group was significantly lower than that of the Controls group $(q<0.05)$ (Supplementary Table S2).

Diversity analysis. Alpha diversity comparisons among three groups. Alpha diversity mainly reflected the richness and diversity of the species in samples. As shown in Fig. 2, indices of Chao 1, Ace, and Shannon of MDD group were significantly higher than those of Follow-up group and Controls group, and the Simpson index was significantly lower in MDD group than others. This showed that the richness and the diversity of gut microbiota in MDD group were significantly higher than those of Follow-up group and Controls group. Indices value of alpha diversity in Follow-up group was significant different from that of MDD group, and there was no significant difference between Follow-up group and Control group. The Alpha diversity of gut microbiota in patients had a tendency to return to normal. The statistics of Alpha diversity index values of each group were shown in Table 2.

Mothur (version v.1.30) software was used to calculate the Alpha diversity index for samples. The larger of the index values of Ace and Chaos1 indices showed the greater number of species in the samples. The larger of the Shannon index value and the smaller of the Simpson index value showed more species categories of samples.

Beta diversity comparisons among three groups. Beta diversity was used to compare the similarity of species diversity among different groups. The binary jaccard algorithm was used to calculate beta diversity. The gut microbiota of MDD group was significantly different with that of controls group, and the gut microbiota within MDD group was more similar ( $\mathrm{R}=0.273, p=0.001)$ (Fig. 3 ).

In addition, the gut microbiota profiles of some patients treated with escitalopram were more similar to those of the control group, but the others' profiles remained closer to those of patients. The other analysis methods employed in this study also produced similar results. The unweighted paired average method (UPGMA) was used in the $\mathrm{R}$ language tool to perform hierarchical clustering of each groups. It found that the gut microbiota of MDD group was significantly different with that of controls group, and the gut microbiota of follow-up group was more similar with that of controls group (Supplementary Figure S1).

The correlation of gut microbiota among the Follow-up group and other groups. Spearman correlation coefficient between samples was calculated to draw the heatmap. The closer of the calculated Spearman correlation coefficient was to 1 , the redder of the color was in the heat map, thus indicating the stronger correlation between two samples. As shown in Fig. 4, the follow-up group could be divided into two subgroups. The gut microbiota profiles of some of the treated patients in the follow-up group remained similar to those in the MDD group, while others' profiles were more similar with those of Controls group. These results suggested that antidepressant drugs could transform the gut microbiota of some patients into that of the control group. LEfSe was used for the quantitative analysis of biomarkers in two subgroups (LDA $>4)$. Several microorganisms could be selected as biomarkers in two subgroups. Gut microbiota of follow-up group 2, the subgroup associated with MDD group, was differently enriched with p_Bacteroidetes, o_Bacteroidales, c_Bacteroidia and g_Prevotella_9. While gut microbiota of follow-up group 1 , the subgroup associated with Controls group, was differently enriched with p_Firmicutes, p_Actinobacteria, f_Lachnospiraceae, f_Bifidobacteriaceae, o_Bifidobacteriales, c_Actinobacteria and g_Bifidobacterium (Supplementary Figure S2).

Functional properties predicted by PICRUSt. The study considered that the profiles of gut microbiota in follow-up group could not completely return to the normal state. The PICRUSt software was used to compare the species composition information obtained from $16 \mathrm{~S}$ sequencing data to infer the functional gene composition between patients and controls. Through the annotation of the KEGG metabolic pathway, it found that there were differences in the metabolic pathways of Transport and catabolism, Nervous system, Glycan biosynthesis and metabolism, Cell motility and Membrane transport between Follow-up group and Controls group $(p<0.05)$ (Fig. 5). In MDD group and Controls group, the major different pathways included Glycan biosynthesis and metabolism, Transport and catabolism, Excretory system, Metabolism of other amino acides, and Nervous system, which were similar with the result of those of Follow-up group and Controls group (Supplementary Figure S3). Meanwhile, only the pathway of Biosynthesis of other secondary metabolites was significantly differences between MDD group and Follow-up group (Supplementary Figure S4). It suggested that the gut microbiota of Follow-up group might still involve in the occurrence of depression. 
a

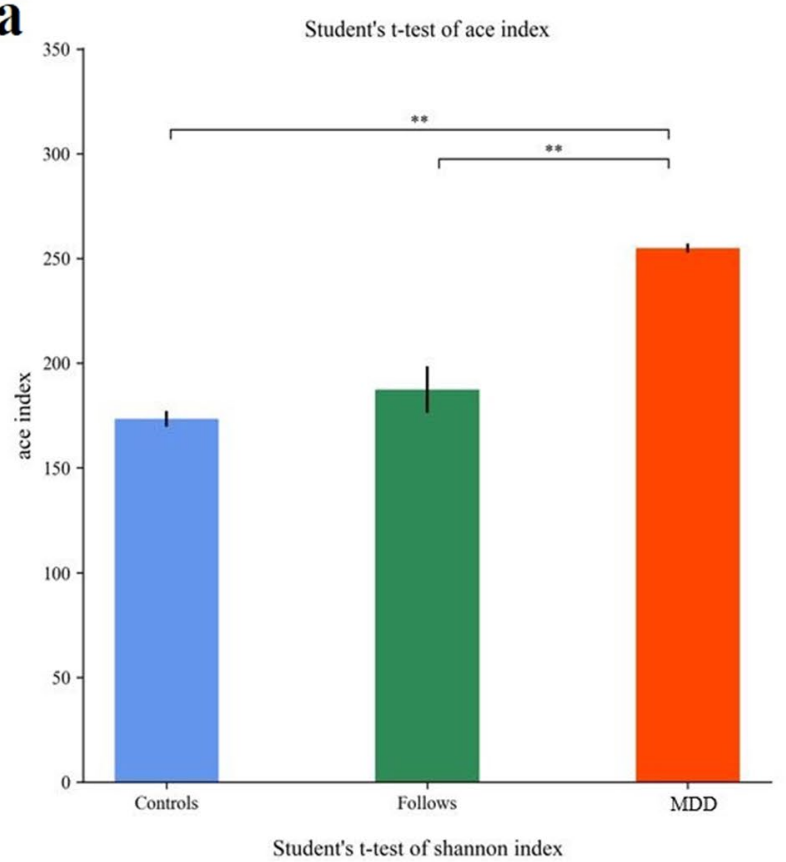

c

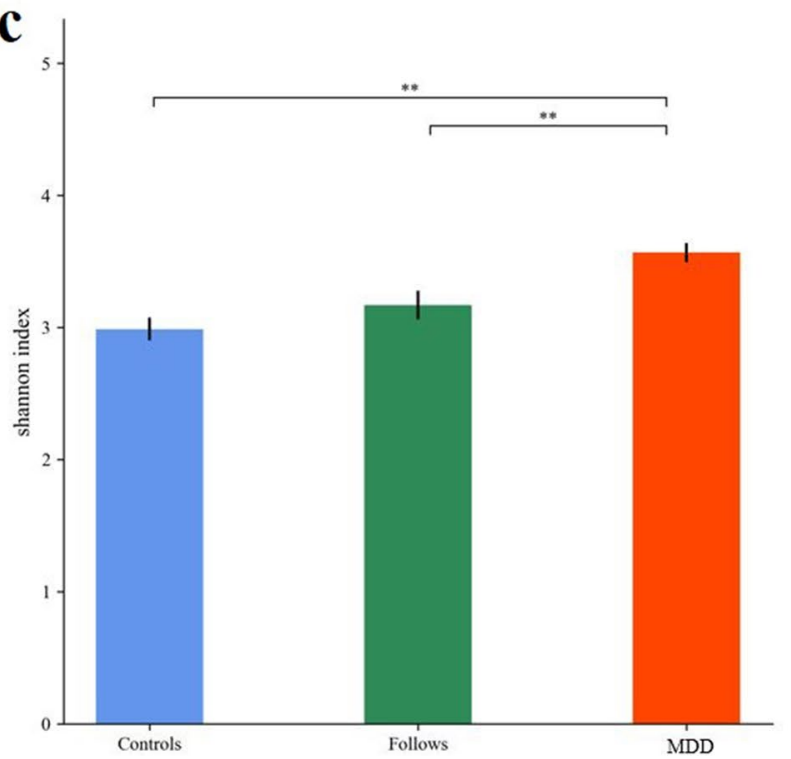

b

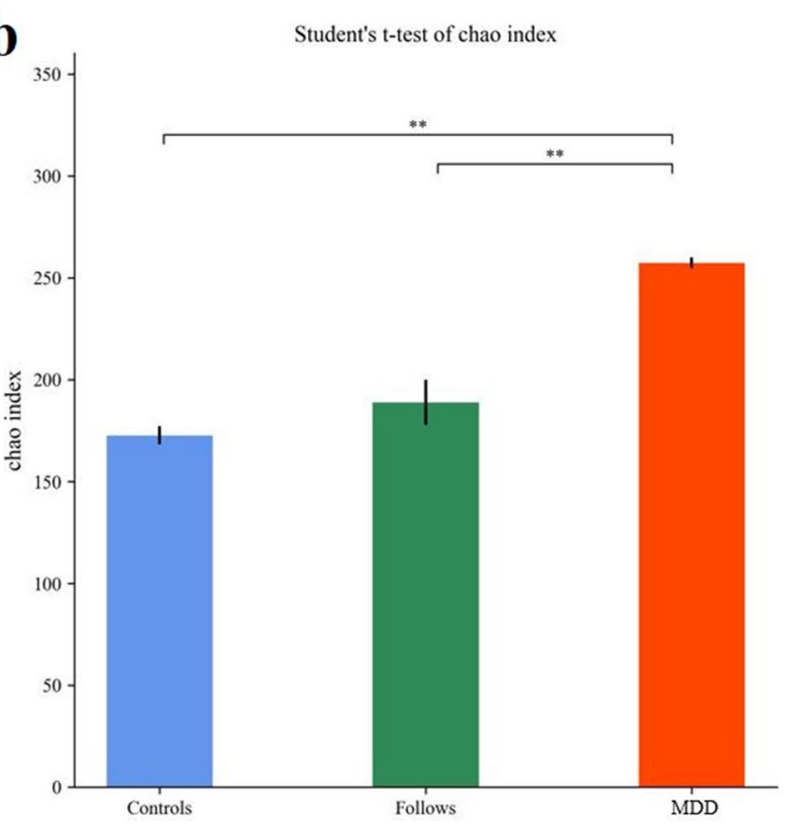

Student's t-test of simpson index

d

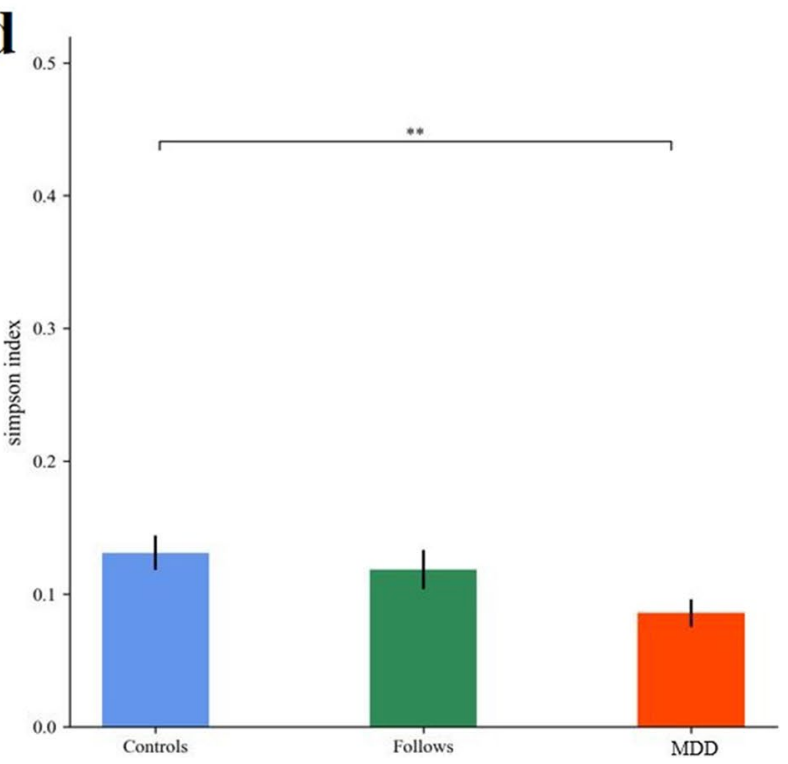

Figure 2. Visualization of Alpha diversity index. (a) Ace index; (b) Chaos1 index; (c) Shannon index; (d) Simpson index. ${ }^{* *}$ means the statistical difference between the two groups, $\mathrm{p}<0.05$.

\begin{tabular}{|l|l|l|l|}
\hline & MDD $($ mean \pm SD $)$ & Follow-up $($ mean \pm SD $)$ & Controls $($ mean \pm SD $)$ \\
\hline Ace & $254.88 \pm 2.30$ & $187.39 \pm 11.09$ & $173.43 \pm 3.80$ \\
\hline Chaos 1 & $257.38 \pm 2.63$ & $188.93 \pm 11.07$ & $172.69 \pm 4.46$ \\
\hline Shannon & $3.57 \pm 0.07$ & $3.17 \pm 0.11$ & $2.99 \pm 0.09$ \\
\hline Simpson & $0.09 \pm 0.01$ & $0.12 \pm 0.01$ & $0.13 \pm 0.01$ \\
\hline
\end{tabular}

Table 2. Richness and diversity index values of MDD, follow-up and controls.

\section{Discussion}

This study demonstrated that the gut microbiota from patients of drug-naive first-episode MDD was significantly different with that of controls, and the composition and structure of the gut microbiota in patients were more similar. This suggested that the occurrence and development of MDD might be associated with specific gut microbiotas. However, the identification results of gut microbiota structure were inconsistent ${ }^{7,20,21}$. In the study 


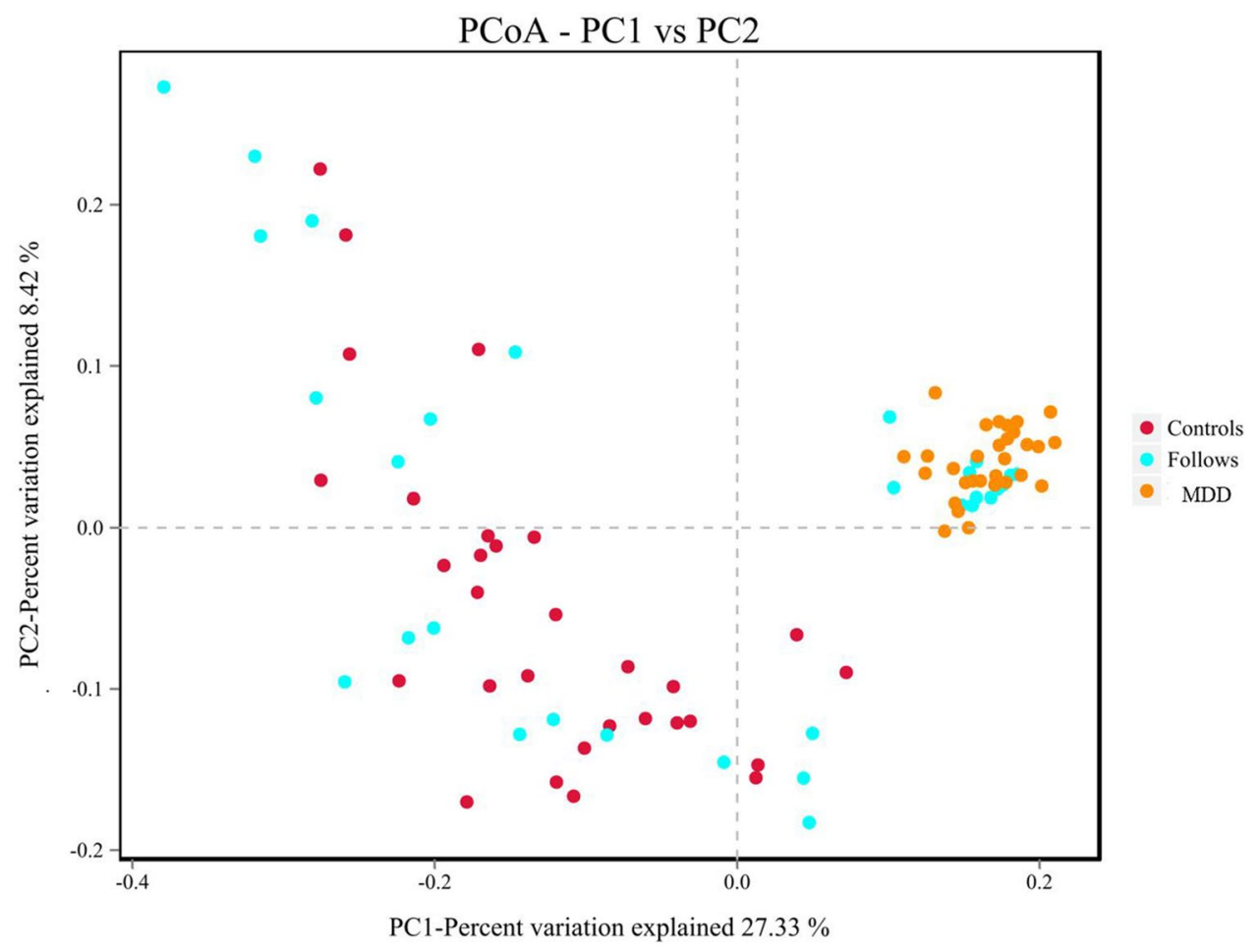

Figure 3. PCoA analysis shows the distribution coordinate diagram of samples: in the graph, the distance between the dots represents the similarity of the samples. Samples with high similarity tend to cluster together. The yellow dots represent the patient group, the blue dots represent the follow-up group, and the red dots represent the control group. The results showed a statistically significant difference among the three groups $(\mathrm{R}=0.273, \mathrm{p}=0.001)$.

of Lin et al., they found the MDD patients had more phylum Firmicutes and less Bacteroidetes ${ }^{7}$. On the contrary, Huang et al. reported that Firmicutes were significantly lower in patients with depression ${ }^{21}$. The inconsistency of the results from different studies may be related to factors including small sample size, different inclusion criteria, course, severity of disease, etc. ${ }^{22}$. However, some studies have shown that transplanting fecal samples from depressed patients could induce depressive symptoms in mice 23,24 . This suggested that a disturbed gut microbiota could be one of the causes of depression.

There is still a lack of effective biomarkers as clinical guidance for the diagnosis and treatment of depression ${ }^{25}$. Several studies have shown that gut microbiota, used as a biomarker, has a good distinguishing effect on depression, AUC value $0.702-0.986^{26-28}$. Gut microbiota profiles can be used to effectively distinguish not only patients from normal population, but also major depressive disorder and bipolar depression, and even evaluate the therapeutic effect as well ${ }^{27,28}$. In this study, the major changed gut microbiota were Bacteroidetes. Monitoring the variation of Bacteroidetes may indicate the prognosis of the disease and the efficacy of the drug, which could be used as a potential biomarker.

In 2011, Manimozhiyan Arumugam et al. proposed the concept of enterotypes ${ }^{29}$. In the sequencing results, they reported that the human gut microbiome could be divided into three robust clusters, including Prevotellaenterotype (enterotype P), Bacteroides-enterotype (enterotype B) and Ruminococcus-enterotype (enterotype R). Different enterotypes have characteristic advantages of respective functional states ${ }^{30}$. For example, enterotype $\mathrm{P}$ can generate more short-chain fatty acids and thus has stronger fermentation ability. Enterobacter type B has a variety of specific enzymes, which can promote the hydrolysis of sugars and proteins in food and improve the absorption by the body ${ }^{31}$. Our study found that the composition of gut microbiota in Follow-up group resembled participants in the control group, but the metabolic function of gut microbiota in Follow-up group was still similar to that of MDD group. We considered that the partial composition of gut microbiota had changed after treatment, but the enterotype was not, so that it still retained abnormal metabolic characteristics. However, we were unable to confirm this hypothesis due to short follow-up period in this study. The Belgian Flemish Gut Flora Project have found that enterotype distribution varied with depression status ${ }^{32}$. Therefore, the future study could explore the relationship between enterotypes and metabolic function in different period of depression, which may help to understand the impact of gut microbiota on the development of MDD.

In addition, the gut microbiota tended to "normal" gut microbiota structure under SSRIs treatment, thus indicating a positive effect of SSRIs on the change of gut microbiota. However, there is currently no consensus on the effect of antidepressants on the gut microbiota. A recent study reported Lachnospiraceae species were more abundant in SSRIs treated mice compared to controls ${ }^{33}$. However, the opposite conclusion was reported in Valles-Colomer et al.'s study ${ }^{32}$. Since gut microbiota is easily affected by a variety of factors, future research 


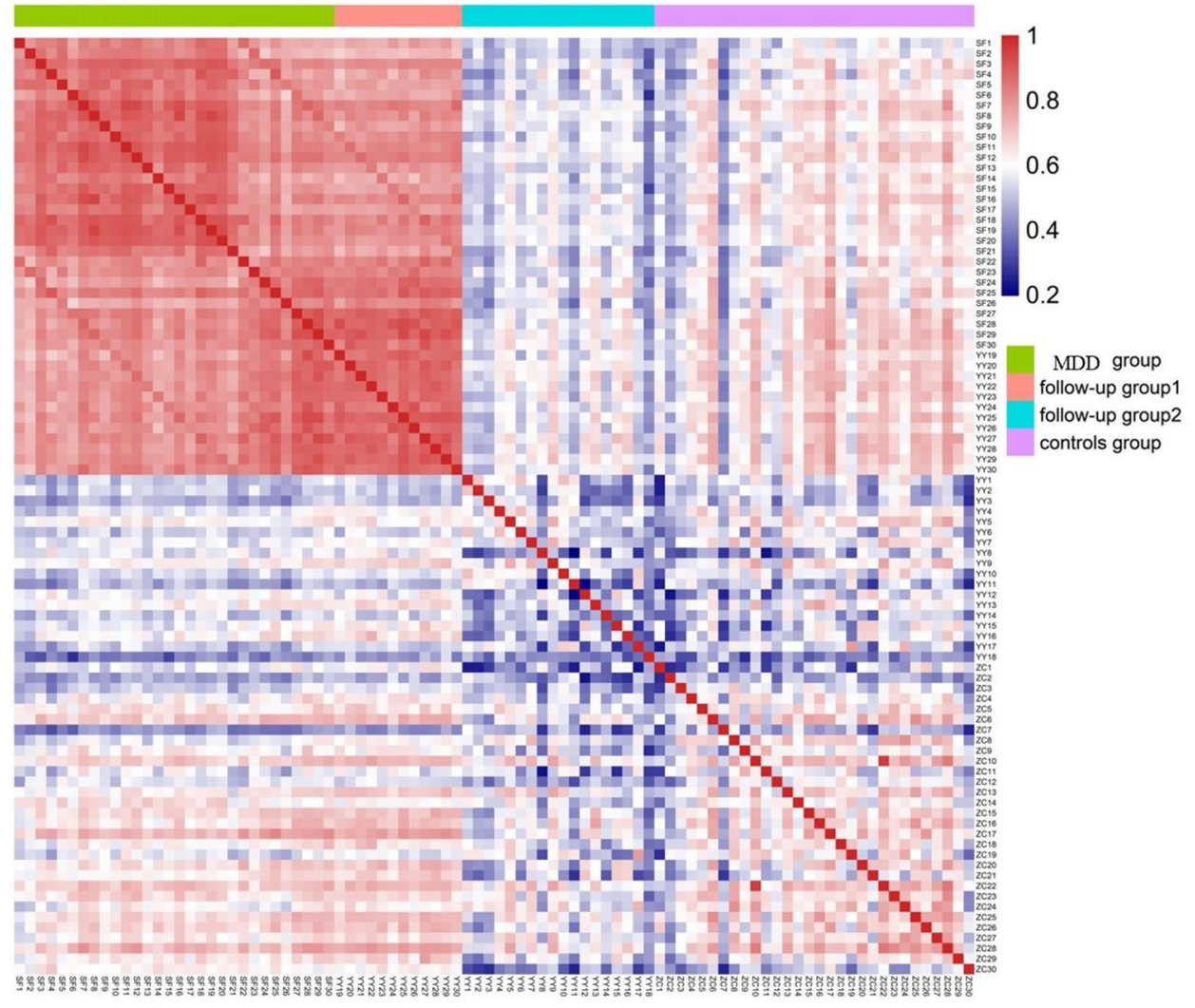

Figure 4. Spearman correlation coefficient heat map between samples. The closer the calculated Spearman correlation coefficient is to 1 , the redder the color in the heat map, indicating the stronger correlation between the two samples. Follow-up group 1 had high correlation with Controls group. Follow-up group 2 had high correlation with MDD group.

Controls

Follow-up

Transport and catabolism

Nervous system

Glycan biosynthesis and metabolism

Cell motility

Membrane transport

Biosynthesis of other secondary metabolites

Excretory system

Cellular community - prokaryotes

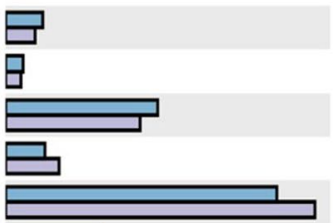

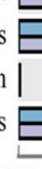$$
0.0
$$

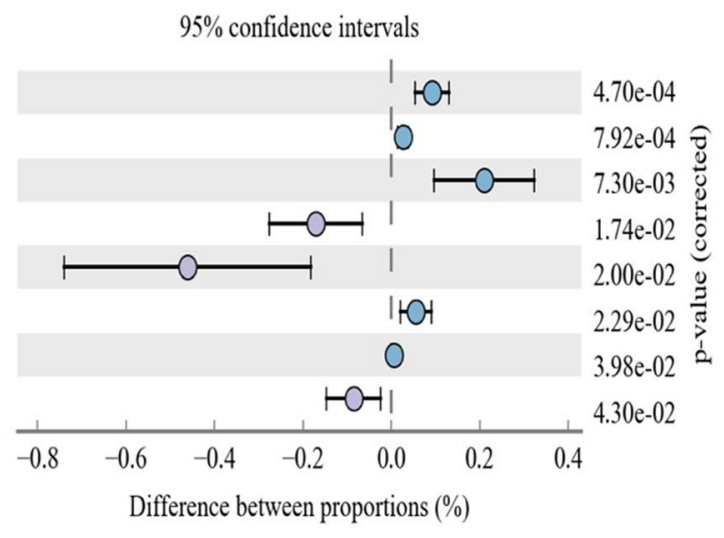

Figure 5. Metabolic pathway analysis between Controls group and Follow-up group. The left side of the figure shows the abundance ratio between the two groups. The middle section shows the proportional variation in functional abundance within the $95 \%$ confidence interval. The p value is on the right.

should pay attention to course of disease, severity, population characteristics and other aspects to further clarify the change of gut microbiota.

The outcomes of current study were limited to the relatively small sample size and short follow-up time. This study was unable to further observe the effects of different antidepressant doses on the composition of gut microbiota. The effect of diet on gut microbiota was also not fully considered. Different diets and microbial combinations have different effects on the physiological function and substance metabolism of the intestinal tract ${ }^{34}$. In the future, we could further expand the sample size for research. Based on the understanding of the changes of gut microbiota, the correlation between gut microbiota and clinical phenotypes could be further elucidated. 
Moreover, metabolomics and proteomics omics technologies are strongly suggested to be employed to explore the relationship between gut microbiota and depression.

\section{Conclusions}

In this study, it found that the gut microbiota of patients with first-episode depression was significantly different with that of Controls group. After escitalopram treatment, gut microbiota diversity of depressive patients tended to return to the normal state. However, there were still several structures and metabolic pathways difference in the gut microbiota between follow-up patients and controls, which might be related to the relapse of depression.

\section{Methods}

Participants. The 60 local subjects, including 30 depressed patients and 30 normal subjects, were recruited in this study. The inclusion criteria for subjects described as follows: (1) age was between 18-65 years old; (2) body mass index (BMI) was between 18 and $28 \mathrm{~kg} / \mathrm{m}^{2}$; (3) no history of treatment with antipsychotic medication; (4) duration of symptoms was between 1 and 24 months; (5) no history of treatment with antidepressant medication; (6) currently in the acute episode with the Hamilton Depression Rating Scale for Depression (HAMD) score $\geq 24$; (7) other mental disorders such as axis I, personality disorder and mental retardation were excluded; (8) psychotropic drugs were never used; (9) diagnosis of depression in MDD group was made by two psychiatrists according to the Mini-International Neuropsychiatric Interview (MINI); (10) in Controls group, a diagnosis of mental disorder was excluded by two psychiatrists according to the MINI, and HAMD-17 score was $<7$; (11) MDD group was defined as Follow-up group after receiving the drug treatment.

In addition, a series of exclusion criteria, based on the previous work, were employed in this study to exclude the factors affecting gut microbiota ${ }^{35}$. Those include: (1) no somatic diseases known to affect the gut microbiota such as inflammatory bowel disease, immune system diseases, diabetes, etc.; (2) without antibiotics, probiotics or microbiological products used in recent 3 months; (3) no history of medical examination or surgery through the gastrointestinal tract in recent 6 months; (4) without obvious changes in dietary habits or the presence of obvious diarrhea, constipation and other symptoms in recent 1 month.

According to the questionnaire, subjects' life events that may affect the mood, such as examinations, unemployment and bereavement during the last six months and the whole research period, were surveyed and recorded. All of subjects in this study were required to sign an informed consent. According to the Helsinki Declaration, the protocol for sample collection and analysis was approved by the Ethics Committee of Peking University Sixth Hospital and Beijing Hospital of Chinese Traditional and Western Medicine.

Sample collection. Fecal samples were obtained from subjects enrolled. Subjects were instructed by staff to discharge feces into a clean container. After defecation, the staff collected $2 \mathrm{~g}$ fecal sample and quickly placed it into a container containing liquid nitrogen. The samples were then frozen at $-80^{\circ} \mathrm{C}$ until analysis.

Treatment. All patients with depression received individualized treatment with escitalopram. The starting dose of escitalopram was $5 \mathrm{mg}$ /day from day 1 -day 7 and increased to $10 \mathrm{mg} /$ day from day 8 . According to the individual response, the dose of escitalopram could be adjusted, and the maximum dose was $20 \mathrm{mg} / \mathrm{day}$. After 4-6 weeks of treatment, the patients were evaluated by HAMD scale. When the scale reduction rate of HAMD was $\geq 50 \%$ compared with baseline, their fecal sample was collected for the second time and recorded as 'Followup group' to be used in the comparison of gut microbiota.

16S rRNA Amplification of V3-V4 region and Illumina Sequencing. Using a PowerSoil DNA kit (MoBio, USA), DNA extraction was performed from $200 \mathrm{mg}$ fecal samples according to manufacturer's instructions. KAPA HiFi HotStart ReadyMix (KAPA, USA) was used to amplify the 16S rRNA (V3-V4) gene marker. Each DNA sample of the bacterial 16S rRNA gene was amplified with primers 341F (GGACTACHVGGGTWT CTAAT) and 805R (ACTCCTACGGGAGGCAGCAG). The primers included a unique 8-nucleotide barcode and an Illumina adapter. Polymerase chain reaction (PCR) conditions were set as follows: initial denaturation at $95{ }^{\circ} \mathrm{C}$ for $5 \mathrm{~min}, 98^{\circ} \mathrm{C}$ denaturation for 20 cycles for $20 \mathrm{~s}, 58{ }^{\circ} \mathrm{C}$ annealing for $30 \mathrm{~s}, 72{ }^{\circ} \mathrm{C}$ extension for $30 \mathrm{~s}$, and $72{ }^{\circ} \mathrm{C}$ final extension for $5 \mathrm{~min}$. The amplicons obtained by PCR were analyzed on $1.5 \%$ agarose gel electrophoresis, and a band of a desired size was purified using a QIAquick gel extraction kit (QIAGEN, Germany). The product was submitted to the second-generation sequencing laboratory of Beijing institute of bioinformatics for sequencing on Illumina HiSeq 2500 platform.

Bioinformatics analysis. The QIIME (Version 1.9.1) software was used to filter and sequence the original sequence to obtain optimized sequences (Tags) ${ }^{36}$. Fragments containing ambiguous characters in the sequence or more than two nucleotide mismatched primers were removed. Usearch (version 10.0) software was used to cluster Tags at a similarity level of $97 \%$ to obtain OTUs ${ }^{37}$. OTUs were annotated based on the Silva (bacterial) and UNITE (fungi) taxonomy databases. QIIME (Version 1.9.1) software was used to generate species richness tables at different taxonomic levels, and $\mathrm{R}$ language tools were used to draw community structure maps at each taxonomic level of the sample. The community structure map of each sample was obtained at the level of taxonomy, class, order, family, genus, species.

In order to identify the difference in microbial community richness between the MDD group and Controls group, the Metastats (URL: http://metastats.cbcb.umd.edu) software was used to perform a T test on the species richness data between two groups to obtain the $p$ value ${ }^{38}$. The $q$ value was obtained by correcting the $p$ value. Species were selected based on $p$ values or $q$ values that caused differences in the composition of the two groups 
of samples. The analysis was performed at the level of phylum, class, order, family, genus, species taxonomy to analyze the significance between groups.

Mothur (version v.1.30, URL: http://www.mothur.org/) software was used to evaluate the Alpha Diversity Index of the samples ${ }^{39}$. The species diversity within a single sample was studied by Alpha Diversity Analysis, and the Ace, Chaol, Shannon, and Simpson indices of each sample at the $97 \%$ similarity level were counted; Beta diversity analysis was performed using QIIME (Version 1.9.1) software. Beta diversity analysis mainly used the binary jaccard algorithm to calculate the distance among samples to obtain the $\beta$ value between samples. Based on the distance matrix obtained from the Beta diversity analysis, PCoA analysis was performed using R language tools to further demonstrate the differences in species diversity among samples ${ }^{40}$. Hierarchical clustering was performed on samples using unweighted paired average method (UPGMA) to determine the similarity of species composition among samples. According to the species abundance table obtained by clustering, spearman correlation coefficient among samples was calculated by Psych package in $\mathrm{R}$ language, and then the heatmap was drawn by $\mathrm{P}$ heatmap package in $\mathrm{R}$ language. The closer of the calculated Spearman correlation coefficient was to 1 , the darker the red shading was in the heat map, thus indicating the stronger correlation of two samples. Then, LEfSe tools (URL: http://huttenhower.sph.harvard.edu/lefse/) were used the Wilcox test function of the R language STATS package to estimate the impact of the abundance of each component (species) on the effect of the difference between components, so that the comparison of two subgroups can be realized to find the species marker (Biomaker) with significant difference in the abundance ${ }^{41}$.

PICRUSt (Version 1.1.4) software was used to compare the species composition information obtained from $16 \mathrm{~S}$ sequencing data to deduce the functional gene composition in the samples, thereby determining the functional differences between different groups ${ }^{42}$. Using the KEGG orthology database (KOs) in the Kyoto Encyclopedia of Genes and Genomics (KEGG) database ${ }^{43}$, the changes in metabolic pathways of functional genes of microbial communities between different groups were evaluated through differential analysis of KEGG metabolic pathways $^{44,45}$.

Statistics analysis. Statistical analysis was performed using SPSS19.0 software. Participants' gender, tobacco and alcohol consumption were expressed in terms of proportional or percentages. Independent t tests, Welch $t$ tests, and White non-parametric $t$ tests were used for continuous variables. Pearson chi-square test or Fisher's exact test were used for classification variables. All significance tests were two-sided tests, and $\mathrm{p}<0.05$ or adjusted $\mathrm{p}<0.05$ was considered statistically significant.

Received: 6 January 2021; Accepted: 9 July 2021

Published online: 21 July 2021

\section{References}

1. Roberts, T. et al. Factors associated with health service utilisation for common mental disorders: a systematic review. BMC Psychiatry 18, 262. https://doi.org/10.1186/s12888-018-1837-1 (2018).

2. Inserra, A., Mastronardi, C. A., Rogers, G., Licinio, J. \& Wong, M. L. Neuroimmunomodulation in major depressive disorder: focus on caspase 1, inducible nitric oxide synthase, and interferon-gamma. Mol. Neurobiol. 56, 4288-4305. https://doi.org/10. 1007/s12035-018-1359-3 (2019).

3. Inserra, A., Rogers, G. B., Licinio, J. \& Wong, M. L. The microbiota-inflammasome hypothesis of major depression. BioEssays 40, e1800027. https://doi.org/10.1002/bies.201800027 (2018).

4. Molina-Torres, G., Rodriguez-Arrastia, M., Roman, P., Sanchez-Labraca, N. \& Cardona, D. Stress and the gut microbiota-brain axis. Behav. Pharmacol. 30, 187-200. https://doi.org/10.1097/FBP.0000000000000478 (2019).

5. Zheng, P. et al. Gut microbiome remodeling induces depressive-like behaviors through a pathway mediated by the host's metabolism. Mol. Psychiatry 21, 786-796. https://doi.org/10.1038/mp.2016.44 (2016).

6. Naseribafrouei, A. et al. Correlation between the human fecal microbiota and depression. Neurogastroenterol. Motil. 26, $1155-1162$. https://doi.org/10.1111/nmo.12378 (2014).

7. Lin, P. et al. Prevotella and Klebsiella proportions in fecal microbial communities are potential characteristic parameters for patients with major depressive disorder. J. Affect. Disord. 207, 300-304. https://doi.org/10.1016/j.jad.2016.09.051 (2017).

8. Jiang, H. et al. Altered fecal microbiota composition in patients with major depressive disorder. Brain Behav. Immun. 48, 186-194. https://doi.org/10.1016/j.bbi.2015.03.016 (2015).

9. Li, B. et al. Metabolite identification in fecal microbiota transplantation mouse livers and combined proteomics with chronic unpredictive mild stress mouse livers. Transl. Psychiatry 8, 34. https://doi.org/10.1038/s41398-017-0078-2 (2018).

10. Luo, Y. et al. Gut microbiota regulates mouse behaviors through glucocorticoid receptor pathway genes in the hippocampus. Transl. Psychiatry 8, 187. https://doi.org/10.1038/s41398-018-0240-5 (2018).

11. Pearson-Leary, J. et al. The gut microbiome regulates the increases in depressive-type behaviors and in inflammatory processes in the ventral hippocampus of stress vulnerable rats. Mol. Psychiatry https://doi.org/10.1038/s41380-019-0380-x (2019).

12. Dale, E., Bang-Andersen, B. \& Sanchez, C. Emerging mechanisms and treatments for depression beyond SSRIs and SNRIs. Biochem. Pharmacol. 95, 81-97. https://doi.org/10.1016/j.bcp.2015.03.011 (2015).

13. Lieb, J. The immunostimulating and antimicrobial properties of lithium and antidepressants. J. Infect. 49, 88-93. https://doi.org/ 10.1016/j.jinf.2004.03.006 (2004).

14. Munoz-Bellido, J. L., Munoz-Criado, S. \& Garcia-Rodriguez, J. A. Antimicrobial activity of psychotropic drugs: selective serotonin reuptake inhibitors. Int. J. Antimicrob. Agents 14, 177-180. https://doi.org/10.1016/s0924-8579(99)00154-5 (2000).

15. Ayaz, M. et al. Sertraline enhances the activity of antimicrobial agents against pathogens of clinical relevance. J. Biol. Res. (Thessalon) 22, 4. https://doi.org/10.1186/s40709-015-0028-1 (2015).

16. Coban, A. Y., Tanriverdi Cayci, Y., Keles Uludag, S. \& Durupinar, B. Investigation of antibacterial activity of sertralin. Mikrobiyol. Bul. 43, 651-656 (2009).

17. Kruszewska, H., Zareba, T. \& Tyski, S. Examination of antimicrobial activity of selected non-antibiotic medicinal preparations. Acta Pol. Pharm. 69, 1368-1371 (2012). 
18. Ramsteijn, A. S., Jasarevic, E., Houwing, D. J., Bale, T. L. \& Olivier, J. D. Antidepressant treatment with fluoxetine during pregnancy and lactation modulates the gut microbiome and metabolome in a rat model relevant to depression. Gut Microbes 11, 735-753. https://doi.org/10.1080/19490976.2019.1705728 (2020).

19. McVey Neufeld, K. A. et al. Oral selective serotonin reuptake inhibitors activate vagus nerve dependent gut-brain signalling. Sci. Rep. 9, 14290. https://doi.org/10.1038/s41598-019-50807-8 (2019).

20. Chen, Z. et al. Comparative metaproteomics analysis shows altered fecal microbiota signatures in patients with major depressive disorder. NeuroReport 29, 417-425. https://doi.org/10.1097/WNR.0000000000000985 (2018).

21. Huang, Y. et al. Possible association of Firmicutes in the gut microbiota of patients with major depressive disorder. Neuropsychiatr. Dis. Treat. 14, 3329-3337. https://doi.org/10.2147/NDT.S188340 (2018).

22. Kelly, J. R. et al. Transferring the blues: Depression-associated gut microbiota induces neurobehavioural changes in the rat. J. Psychiatr. Res. 82, 109-118. https://doi.org/10.1016/j.jpsychires.2016.07.019 (2016).

23. Xu, Z. et al. Fecal microbiota transplantation from healthy donors reduced alcohol-induced anxiety and depression in an animal model of chronic alcohol exposure. Chin. J. Physiol. 61, 360-371. https://doi.org/10.4077/CJP.2018.BAH633 (2018).

24. Kurokawa, S. et al. The effect of fecal microbiota transplantation on psychiatric symptoms among patients with irritable bowel syndrome, functional diarrhea and functional constipation: An open-label observational study. J. Affect. Disord. 235, 506-512. https://doi.org/10.1016/j.jad.2018.04.038 (2018).

25. Buckman, J. E. J. et al. Risk factors for relapse and recurrence of depression in adults and how they operate: A four-phase systematic review and meta-synthesis. Clin. Psychol. Rev. 64, 13-38. https://doi.org/10.1016/j.cpr.2018.07.005 (2018).

26. Lai, W. T. et al. Shotgun metagenomics reveals both taxonomic and tryptophan pathway differences of gut microbiota in major depressive disorder patients. Psychol. Med. 51, 90-101. https://doi.org/10.1017/S0033291719003027 (2021).

27. Hu, S. et al. Gut microbiota changes in patients with bipolar depression. Adv. Sci. (Weinh) 6, 1900752. https://doi.org/10.1002/ advs.201900752 (2019).

28. Zheng, P. et al. Gut microbial signatures can discriminate unipolar from bipolar depression. Adv. Sci. (Weinh) 7, 1902862. https:// doi.org/10.1002/advs.201902862 (2020).

29. Arumugam, M. et al. Enterotypes of the human gut microbiome. Nature 473, 174-180. https://doi.org/10.1038/nature09944 (2011).

30. Chen, T. et al. Fiber-utilizing capacity varies in Prevotella- versus Bacteroides-dominated gut microbiota. Sci. Rep. 7, 2594. https:// doi.org/10.1038/s41598-017-02995-4 (2017).

31. Vieira-Silva, S. et al. Species-function relationships shape ecological properties of the human gut microbiome. Nat. Microbiol. 1, 16088. https://doi.org/10.1038/nmicrobiol.2016.88 (2016).

32. Valles-Colomer, M. et al. The neuroactive potential of the human gut microbiota in quality of life and depression. Nat. Microbiol. 4, 623-632. https://doi.org/10.1038/s41564-018-0337-x (2019).

33. Lyte, M., Daniels, K. M. \& Schmitz-Esser, S. Fluoxetine-induced alteration of murine gut microbial community structure: evidence for a microbial endocrinology-based mechanism of action responsible for fluoxetine-induced side effects. PeerJ 7, e6199. https:// doi.org/10.7717/peerj.6199 (2019).

34. Dey, N. et al. Regulators of gut motility revealed by a gnotobiotic model of diet-microbiome interactions related to travel. Cell 163, 95-107. https://doi.org/10.1016/j.cell.2015.08.059 (2015)

35. Shen, Y. et al. Analysis of gut microbiota diversity and auxiliary diagnosis as a biomarker in patients with schizophrenia: A crosssectional study. Schizophr. Res. 197, 470-477. https://doi.org/10.1016/j.schres.2018.01.002 (2018).

36. Caporaso, J. G. et al. QIIME allows analysis of high-throughput community sequencing data. Nat. Methods 7, 335-336. https:// doi.org/10.1038/nmeth.f.303 (2010).

37. Edgar, R. C. UPARSE: highly accurate OTU sequences from microbial amplicon reads. Nat. Methods 10, 996-998. https://doi.org/ 10.1038/nmeth.2604 (2013).

38. White, J. R., Nagarajan, N. \& Pop, M. Statistical methods for detecting differentially abundant features in clinical metagenomic samples. PLoS Comput. Biol. 5, e1000352. https://doi.org/10.1371/journal.pcbi.1000352 (2009).

39. Grice, E. A. et al. Topographical and temporal diversity of the human skin microbiome. Science 324, 1190-1192. https://doi.org/ 10.1126/science.1171700 (2009).

40. Sakaki, T., Takeshima, T., Tominaga, M., Hashimoto, H. \& Kawaguchi, S. Recurrence of ICA-PCoA aneurysms after neck clipping. J. Neurosurg. 80, 58-63. https://doi.org/10.3171/jns.1994.80.1.0058 (1994).

41. Segata, N. et al. Metagenomic biomarker discovery and explanation. Genome Biol. 12, R60. https://doi.org/10.1186/gb-2011-12-6r60 (2011).

42. Parks, D. H., Tyson, G. W., Hugenholtz, P. \& Beiko, R. G. STAMP: statistical analysis of taxonomic and functional profiles. Bioinformatics 30, 3123-3124. https://doi.org/10.1093/bioinformatics/btu494 (2014).

43. Kanehisa, M. et al. Data, information, knowledge and principle: back to metabolism in KEGG. Nucleic Acids Res. 42, D199-205. https://doi.org/10.1093/nar/gkt1076 (2014)

44. Langille, M. G. et al. Predictive functional profiling of microbial communities using $16 \mathrm{~S}$ rRNA marker gene sequences. Nat. Biotechnol. 31, 814-821. https://doi.org/10.1038/nbt.2676 (2013).

45. Kanehisa, M. \& Sato, Y. KEGG Mapper for inferring cellular functions from protein sequences. Protein Sci. 29, 28-35. https://doi. org/10.1002/pro.3711(2020)

\section{Acknowledgements}

We thank all the subjects who took part in this study. Thank XXX, ZYL and YCH for their support in this study.

\section{Author contributions}

Y.S., X.Y., J.Y.G. and Y.L. designed the study and wrote the protocol. X.Y. and J.Y.G. managed the literature searches and analyses. Y.S. undertook the statistical analysis, and Y.S., X.Y. and G.F.L. wrote the first draft of the manuscript. All authors contributed to and have approved the final manuscript.

\section{Funding}

This study was supported by Beijing Association for Science and Technology Jinqiao Seed Fund Project (no. JQ18005). The role of funding body was in the design and implementation of study.

\section{Competing interests}

The authors declare no competing interests.

Additional information

Supplementary Information The online version contains supplementary material available at https://doi.org/ 10.1038/s41598-021-94481-1. 
Correspondence and requests for materials should be addressed to J.G. or Y.L.

Reprints and permissions information is available at www.nature.com/reprints.

Publisher's note Springer Nature remains neutral with regard to jurisdictional claims in published maps and institutional affiliations.

(c) (i) Open Access This article is licensed under a Creative Commons Attribution 4.0 International cc) License, which permits use, sharing, adaptation, distribution and reproduction in any medium or format, as long as you give appropriate credit to the original author(s) and the source, provide a link to the Creative Commons licence, and indicate if changes were made. The images or other third party material in this article are included in the article's Creative Commons licence, unless indicated otherwise in a credit line to the material. If material is not included in the article's Creative Commons licence and your intended use is not permitted by statutory regulation or exceeds the permitted use, you will need to obtain permission directly from the copyright holder. To view a copy of this licence, visit http://creativecommons.org/licenses/by/4.0/.

(C) The Author(s) 2021 